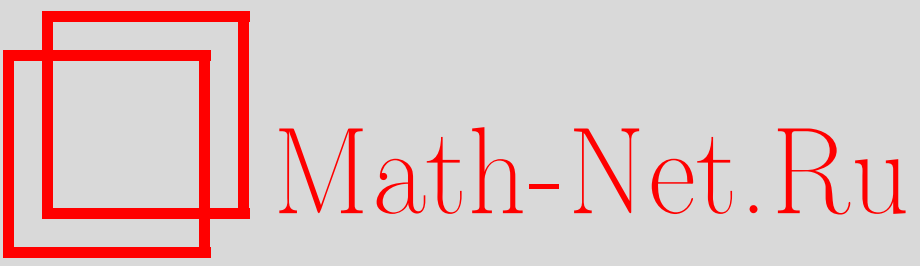

К. Клейн, Решение Керра на частично вырожденных гиперэллиптических римановых поверхностях, ТМФ, 2003, том 137, номер 2, 193-200

DOI: https://doi.org/10.4213/tmf264

Использование Общероссийского математического портала Math-Net.Ru подразумевает, что вы прочитали и согласны с пользовательским соглашением

http://www . mathnet.ru/rus/agreement

Параметры загрузки:

IP: 54.224 .60 .19

26 апреля 2023 г., 14:52:52 


\section{РЕШЕНИЕ КЕРРА НА ЧАСТИЧНО ВЫРОЖДЕННЫХ ГИПЕРЭЛЛИПТИЧЕСКИХ РИМАНОВЫХ ПОВЕРХНОСТЯХ}

Решение Керра для вращающейся черной дыры можно построить как "солитонный” предел тета-функциональных решений Короткина уравнений Эрнста на поверхности рода $g=2$. Показано, что решение Керра можно также получить на частично вырожденной гиперэллиптической римановой поверхности произвольного четного рода.

Ключевые слова: черные дыры, римановы поверхности, солитонный предел.

\section{1. ВВЕДЕНИЕ}

Физическая значимость стационарных аксиально-симметричных уравнений Эйнштейна определяется тем, что их решения могут описывать гравитационное поле черных дыр и звезд в термодинамическом равновесии. Наиболее известным решением этих уравнений является решение Керра [1], которое описывает внешнее гравитационное поле врашаюшейся черной дыры. Уравнения Эйнштейна в вакууме эквивалентны уравнениям Эрнста [2]

$$
\operatorname{Re} \mathcal{E}\left(\mathcal{E}_{\rho \rho}+\frac{1}{\rho} \mathcal{E}_{\rho}+\mathcal{E}_{\zeta \zeta}\right)=\mathcal{E}_{\rho}^{2}+\mathcal{E}_{\zeta}^{2}
$$

для комплекснозначного потенциала Эрнста $\mathcal{E}$, зависящего от двух цилиндрических координат $\rho, \zeta$. Их полная интегрируемость была показана Мезоном [3], а также Белинским и Захаровым [4]. В рамках метода обратной задачи рассеяния решение Керра соответствует двухсолитонному решению уравнения Эрнста. Тета-функциональные решения уравнения Эрнста были даны Короткиным [5], [6] на гиперэллиптической римановой поверхности $\mathcal{L}$ рода $g$, соответствуюшей спектральной кривой

$$
\mu^{2}=(K-\xi)(K-\bar{\xi}) \prod_{i=1}^{g}\left(K-E_{i}\right)\left(K-F_{i}\right)
$$

где $\xi=\zeta-i \rho$. Точки ветвления $E_{i}$ и $F_{i}$ не зависят от физических координат и подчинены условию вешественности $E_{i}=\bar{F}_{i}$ или $E_{i}, F_{i} \in \mathbb{R}$. Соответствуюшие решения уравнений Эрнста можно записать в терминах тета-функций с характеристиками $[\mathbf{p}, \mathbf{q}]$ на $\mathcal{L}$ в

* LUTh, Observatoire de Paris, 92195 Meudon Cedex, France; MPI für Physik, Föhringer Ring 6, 80805 München, Germany. E-mail: klein@mppmu.mpg.de 
виде [6]

$$
\mathcal{E}=\frac{\Theta_{\mathbf{p q}}\left(\int_{\xi}^{\infty^{+}}\right)}{\Theta_{\mathbf{p q}}\left(\int_{\xi}^{\infty^{-}}\right)},
$$

где $\int_{b}^{a}$ - сокрашенное обозначение для разности отображений Абеля на поверхности $\mathcal{L}$ между точками $a$ и $b$. Постоянные (по отношению к физическим координатам) векторы $\mathbf{p}$ и q должны удовлетворять условию вешественности $\operatorname{Re}(\Pi \mathbf{p}+2 \pi i \mathbf{q})=0$, где П-матрица $b$-периодов. Символы $\infty^{+}$и $\infty^{-}$используются для бесконечных точек на различных листах кривой $\mathcal{L}$, а именно $\mu / K^{g+1} \rightarrow \pm 1$ при $K \rightarrow \infty^{ \pm}$.

Сушественное различие между решением (3) уравнения Эрнста и, например, конечнозонными решениями уравнений Кортевега-де Фриза состоит в зависимости некоторых точек ветвления гиперэллиптической поверхности от физических координат. Поэтому решения (3) определяются на семействе $\mathcal{L}(\xi, \bar{\xi})$ римановых поверхностей и, вообше говоря, не являются периодическими или квазипериодическими, но являются асимптотически плоскими. В работе [7] было показано, что поверхности с точками ветвления, подчиненными условиям $E_{i}=\bar{F}_{i}, i=1, \ldots, g$, и $E_{i}=-F_{g+1-i}, i=1, \ldots, g / 2$, допускают решения с экваториальной симметрией $\mathcal{E}(-\zeta)=\overline{\mathcal{E}}(\zeta)$.

Как показано в [5], решение Керра можно получить в стандартном солитонном пределе на римановой поверхности рода $g=2$, где точки ветвления $E_{i}, F_{i}$ попарно совпадают. Потенциал Эрнста для решения Керра можно, таким образом, записать в терминах элементарных функций,

$$
\mathcal{E}=\frac{e^{-i \varphi} r_{1}+e^{i \varphi} r_{2}-2 \alpha}{e^{-i \varphi} r_{1}+e^{i \varphi} r_{2}+2 \alpha}
$$

где

$$
r_{1}^{2}=(\zeta+\alpha)^{2}+\rho^{2}, \quad r_{2}^{2}=(\zeta-\alpha)^{2}+\rho^{2}, \quad \alpha=m \cos \varphi .
$$

Масса Арновитта-Дезера-Мизнера пространства-времени равняется $m$, а момент импульса $J=m^{2} \sin \varphi$. В используемых координатах Вейля горизонт черной дыры располагается на оси между $-\alpha$ и $\alpha$. В пределе $\varphi=0$ решение становится статическим и сферически-симметричным решением Шварцшильда; в пределе экстремального решения Керра $\varphi=\pi / 2$ горизонт вырождается.

В настояшей работе мы ставим вопрос о том, какие решения связаны с частично вырожденными римановыми поверхностями высших родов. Этот вопрос важен для построения точных решений уравнений Эйнштейна, описываюших системы “черная дыра-материя". Пусть $\tilde{\mathcal{L}}$-поверхность, заданная спектральной кривой четного рода $g+2$ указанного вида. Рассмотрим предел, в котором разрезы $g+1$ и $g+2$ стягиваются соответственно в точки $-\alpha$ и $\alpha$ на вешественной оси. Мы покажем, что решения (3), которые в данном пределе задаются в терминах тета-функций соответствуюшей римановой поверхности рода $g$, эквивалентны решению Керра при подходяшим образом выбранной характеристике. Доказательство этой теоремы проводится с использованием алгебраического представления решений (3) (см., например, [8]) и теоремы Абеля.

В разделе 2 собраны некоторые полезные факты о тета-функциональных решениях уравнений Эрнста. В разделе 3 рассматривается стандартное вырождение поверхности рода $g=2$, которое в пределе позволяет получить из решения (3) решение Керра (4) результат, обобшаемый на частично вырожденные поверхности высших родов. Результаты кратко суммируются в разделе 4 . 


\section{2. ГИПЕРЭЛЛИПТИЧЕСКИЕ РЕШЕНИЯ УРАВНЕНИЙ ЭРНСТА}

Рассмотрим вешественную гиперэллиптическую риманову поверхность $\mathcal{L}$ рода $g$, заданную соотношением (2) и условием вешественности $E_{i}=\bar{F}_{i} \vee E_{i}, F_{i} \in \mathbb{R}$, где $\xi=\zeta-$ $i \rho, \zeta, \rho \in \mathbb{R}$, и $E_{i}$ и $F_{i}$ не зависят от физических координат. Введем канонический базис циклов на поверхности $\mathcal{L}: a$-циклы выбираются так, что они охватывают разрезы $\left[E_{i}, F_{i}\right]$, а все $b$-циклы начинаются на разрезах $[\xi, \bar{\xi}]$. Базисные голоморфные дифференциалы нормированы условиями

$$
\int_{a_{i}} d \omega_{j}=2 \pi i \delta_{i j}
$$

а матрица П их $b$-периодов определяется как

$$
\Pi_{i j}=\int_{b_{i}} d \omega_{j}
$$

Отображение Абеля с базисной точкой $\xi$ обозначается как $\int_{\xi}^{P}=\int_{\xi}^{P} d \omega$. Дифференциал третьего рода с полюсами первого порядка в точках $A$ и $B$ с соответствуюшими вычетами +1 и -1 обозначается как $d \omega_{A B}$. Тета-функция с характеристиками, соответствующая кривой $\mathcal{L}$, имеет вид

$$
\Theta_{\mathbf{p q}}(\mathbf{z} \mid \Pi)=\sum_{\mathbf{m} \in \mathbb{Z} g} \exp \left\{\frac{1}{2}\langle\Pi(\mathbf{p}+\mathbf{m}), \mathbf{p}+\mathbf{m}\rangle+\langle\mathbf{p}+\mathbf{m}, \mathbf{z}+2 \pi i \mathbf{q}\rangle\right\},
$$

где $\mathbf{z} \in \mathbb{C}^{g}$ - аргумент, $\mathbf{p}, \mathbf{q} \in \mathbb{C}^{g}$ - векторы характеристик, и $\langle\cdot, \cdot\rangle$ обозначает скалярное произведение. С точностью до экспоненциального множителя тета-функция с характеристиками равна тета-функции с нулевыми характеристиками (обозначаемой как $\Theta)$ и сдвинутым аргументом:

$$
\Theta_{\mathbf{p q}}(\mathbf{z} \mid \Pi)=\Theta(\mathbf{z}+\Pi \mathbf{p}+\mathbf{q}) \exp \{\pi i\langle\Pi \mathbf{p}, \mathbf{p}\rangle+\langle\mathbf{p}, \mathbf{z}+2 \pi i \mathbf{q}\rangle\} .
$$

В дальнейшем $K^{+}$будет обозначать точку, принадлежащую верхнему листу поверхности $\mathcal{L}$ и имеющую проекцию $K$ на $\mathbb{C} P^{1}$, а $K^{-}-$точку, имеющую ту же проекцию на $\mathbb{C} P^{1}$, но принадлежащую нижнему листу.

Тета-функциональные решения уравнений Эрнста (1), соответствующие алгебраической кривой (2), даются в следуюшем предложении, принадлежашем Короткину [5].

ПрЕДЛОЖЕНИЕ 1. Пусть $\Theta_{\mathbf{p q}}-$ тета-функиия на поверхности (2) с постоянной $($ по $\xi, \bar{\xi})$ характеристикой, подчиненной условию вещественности $\operatorname{Re}(\Pi p+$ $2 i \pi \mathbf{q})=0$, и пусть $\Theta_{\mathbf{p q}}\left(\int_{\xi}^{\infty^{-}}\right) \neq 0$. Тогда

$$
\mathcal{E}=\frac{\Theta_{\mathbf{p q}}\left(\int_{\xi}^{\infty^{+}}\right)}{\Theta_{\mathbf{p q}}\left(\int_{\xi}^{\infty^{-}}\right)}
$$

является решением уравнения Эрнста.

Доказательство этого предложения можно найти в работе [6]. Заметим, что здесь предполагается, что пути интегрирования в числителе и знаменателе имеют одну и ту же проекцию на $\mathbb{C} P^{1}$, т.е. $\int_{\xi}^{\infty^{+}}=-\int_{\xi}^{\infty^{-}}$. 
В астрофизических приложениях важную роль играет экваториальная симметрия, т.е. симметрия отражения в плоскости $\zeta=0$. В дальнейшем мы будем рассматривать только решения с этим свойством, т.е.

$$
\mathcal{E}(-\zeta)=\overline{\mathcal{E}}(\zeta)
$$

В работе [7] было показано, что решения с этой симметрией можно получить на поверхности четного рода со свойством $\mu(K,-\zeta)=\mu(-K, \zeta)$. Здесь мы ограничимся случаем поверхности с $E_{i}=\bar{F}_{i}, i=1, \ldots, g$, и $E_{i}=-F_{g+1-i}, i=1, \ldots, g / 2$.

Иногда удобно использовать алгебраическую формулировку потенциала Эрнста (8). Используя соотношение (7), перепишем потенциал в терминах тета-функций с нулевой характеристикой:

$$
\mathcal{E}=\frac{\Theta\left(\int_{\xi}^{\infty^{+}}+\Pi \mathbf{p}+\mathbf{q}\right)}{\Theta\left(\int_{\xi}^{\infty^{-}}+\Pi \mathbf{p}+\mathbf{q}\right)} \exp \left\{\left\langle\mathbf{p}, \int_{\infty^{-}}^{\infty^{+}}\right\rangle\right\} .
$$

Определим дивизоры $X$ и $D$ степени $g$ как решение задачи обрашения Якоби

$$
\int_{D}^{X}=\Pi \mathbf{p}+\mathbf{q}
$$

где $D$ выбрано так, что $\int_{\xi}^{D}=\mathbf{K}$ - вектор Римана, который можно выразить в терминах полупериодов на гиперэллиптических поверхностях (см., например, [9]; обозначения для интегралов между дивизорами такие же, как в [10]). Теорема обрашения Якоби гарантирует, что дивизор $X$ корректно определен. Он однозначно определяется формулой (11), за исключением того случая, когда выражение Пр $+2 \pi i \mathbf{q}$ является специальным - случай, который мы всегда исключаем в дальнейшем. Используя дивизор $X$, можно записать потенциал (10) в алгебраическом виде, т.е. в виде, не содержашем тета-функций:

$$
\ln \mathcal{E}=\int_{D}^{X} d \omega_{\infty^{-} \infty^{+}}+\left\langle\mathbf{p}, \int_{\infty^{-}}^{\infty^{+}}\right\rangle
$$

В дальнейшем нам потребуется следуюшая теорема Абеля: если $Y$ и $Z$ являются дивизорами, подчиненными условию

$$
\int_{Z}^{Y}=0
$$

то существует мероморфная функция $R$ на $\mathcal{L}$ (являющаяся рациональной функцией в фундаментальном многоугольнике, образованном разрезанной поверхностью) с полюсами только в $Y$ и нулями только в $Z$. Следствием этой теоремы является тождество

$$
\int_{Z}^{Y} d \omega_{A B}=\ln \frac{R(A)}{R(B)}
$$




\section{3. РЕШЕНИЕ КЕРРА НА ЧАСТИЧНО ВЫРОЖДЕННЫХ ПОВЕРХНОСТЯХ}

В данном разделе мы выясним, как решение Керра возникает в предельном случае подходяших вырождений римановой поверхности $\mathcal{L}$. Для этого рассмотрим риманову поверхность $\tilde{\mathcal{L}}$ четного рода $g+2$ вида $(2)$. Пусть выполнены условия вещественности из предыдушего раздела, а также выполнены условия симметрии $E_{i}=-F_{g+1-i}$, $i=1, \ldots, g / 2$, и $E_{g+1}=-F_{g+2}$. Рассмотрим предел $E_{g+j} \rightarrow F_{g+j}=K_{j}, j=1,2$, при $K_{1}=-K_{2}=-\alpha$.

В этом пределе риманова поверхность вырождается и все функции в формуле (8) можно выразить в терминах функций и дифференциалов, определенных на поверхности $\mathcal{L}$, заданной уравнением (2) (см. [11]). Голоморфные дифференциалы $d \widetilde{\omega}$ на $\tilde{\mathcal{L}}$ становятся дифференциалами на $\mathcal{L}$ :

$$
\begin{aligned}
d \widetilde{\omega}_{i} & =d \omega_{i}, \quad i=1, \ldots, g, \\
d \widetilde{\omega}_{g+j} & =d \omega_{E_{g+j}^{-} E_{g+j}^{+}}, \quad j=1,2 .
\end{aligned}
$$

Дифференциалы третьего рода на $\tilde{\mathcal{L}}$ принимают вид просто соответствуюших дифференциалов третьего рода на $\mathcal{L}$. Компоненты $\Pi_{(g+1)(g+1)}$ и $\Pi_{(g+2)(g+2)}$ матрицы $b$-периодов расходятся, тогда как все остальные компоненты остаются в пределе конечными. При таких соотношениях потеншиал (8) можно полностью выразить в терминах функций, определенных на $\mathcal{L}$ в рассматриваемом пределе.

3.1. Случай $g=0$. Поучительно сначала рассмотреть случай, когда $g=0$, аналогично [5]. Имеем следующее

ПРЕДЛОЖЕНИЕ 2. Пусть характеристика тета-функиий в (8) задана как

$$
\left[\begin{array}{cc}
\frac{1}{2} & \frac{1}{2} \\
-\frac{1}{4}+\frac{\ln a_{1}}{2 \pi i} & \frac{1}{4}+\frac{\ln a_{2}}{2 \pi i}
\end{array}\right],
$$

где $a_{1}=1 / a_{2}=-\operatorname{ctg}(\varphi / 2)$ при $0<\varphi<\pi / 2$. Тогда в пределе $E_{1} \rightarrow F_{1}=-\alpha$, $E_{2} \rightarrow F_{2}=\alpha$ решение тождественно решению Керра и потенциал Эрнста имеет вид (4).

ДОкАЗАТЕЛЬСтво. В пределе $E_{i} \rightarrow F_{i}, i=1,2$, все функции в (8) задаются на римановой поверхности $\mathcal{L}_{0}$ рода $g=0$, заданной как $\mu_{0}^{2}=(K-\xi)(K-\bar{\xi})$. Отсюда следует, что их можно выразить через элементарные функции. Голоморфные дифференциалы становятся дифференциалами третьего рода $d \omega_{E_{i}^{-} E_{i}^{+}}$. Поскольку $b$-периоды $\Pi_{11}$ и $\Pi_{22}$ расходятся, для потенциала Эрнста получаем

$$
\mathcal{E}=\frac{1+a_{1} a_{2} e^{\omega_{1}\left(\infty^{+}\right)+\omega_{2}\left(\infty^{+}\right)}-i e^{-P}\left(a_{1} e^{\omega_{1}\left(\infty^{+}\right)}-a_{2} e^{\omega_{2}\left(\infty^{+}\right)}\right)}{e^{\omega_{1}\left(\infty^{+}\right)+\omega_{2}\left(\infty^{+}\right)}+a_{1} a_{2}-i e^{-P}\left(a_{1} e^{\omega_{2}\left(\infty^{+}\right)}-a_{2} e^{\omega_{1}\left(\infty^{+}\right)}\right)}
$$

где

$$
P=-\frac{1}{2} \Pi_{12}=\int_{\bar{\xi}}^{K_{1}} d \omega_{2}==\ln \frac{\left(E_{1}-E_{2}+r_{1}-r_{2}\right)\left(\bar{\xi}-E_{2}+r_{2}\right)}{\left(E_{1}-E_{2}+r_{1}+r_{2}\right)\left(\bar{\xi}-E_{2}-r_{2}\right)}
$$

и

$$
\omega_{i}\left(\infty^{+}\right)=\ln \frac{E_{i}-\zeta+r_{i}}{-i \rho}
$$


Записывая потенциал Эрнста в виде

$$
\mathcal{E}=\frac{\mathcal{G}-1}{\mathcal{G}+1}
$$

получаем

$$
\mathcal{G}=\frac{1+a_{1} a_{2}}{1-a_{1} a_{2}-i\left(a_{1}+a_{2}\right)} \frac{r_{1}-r_{2}}{E_{1}-E_{2}}+\frac{i\left(a_{1}-a_{2}\right)}{1-a_{1} a_{2}-i\left(a_{1}+a_{2}\right)} \frac{r_{1}+r_{2}}{E_{1}-E_{2}},
$$

что с учетом определения $a_{i}$ является решением Керра в виде (4). Этим завершается доказательство.

3.2. Случай $g>0$. Рассмотрим характеристику

$$
\left[\begin{array}{ccccc}
0 & \cdots & 0 & \frac{1}{2} & \frac{1}{2} \\
\frac{1}{2} & \cdots & \frac{1}{2} & -\frac{1}{4}+\frac{\ln a_{1}}{2 \pi i} & \frac{1}{4}+\frac{\ln a_{2}}{2 \pi i}
\end{array}\right],
$$

где $a_{i}$ определены как в предложении 2. Поскольку $b$-периоды $\Pi_{(g+1)(g+1)}$ и $\Pi_{(g+2)(g+2)}$ расходятся, тета-функция $\widetilde{\Theta}_{\tilde{\mathbf{p}}} \tilde{\mathbf{q}}(\tilde{\mathbf{x}})$ в рассматриваемом пределе пропорциональна выражению

$$
\begin{aligned}
\Theta_{\mathbf{p q}}(x+ & \left.\int_{E_{1}^{-}}^{E_{2}^{+}}\right)-a_{1} a_{2} \Theta_{\mathbf{p q}}\left(x-\int_{E_{1}^{-}}^{E_{2}^{+}}\right) e^{x_{g+1}+x_{g+2}}- \\
& -i e^{-P}\left(a_{1} \Theta_{\mathbf{p q}}\left(x+\int_{E_{1}^{+}}^{E_{2}^{+}}\right) e^{x_{g+1}}-a_{2} \Theta_{\mathbf{p q}}\left(x-\int_{E_{1}^{+}}^{E_{2}^{+}}\right) e^{x_{g+2}}\right),
\end{aligned}
$$

где $P=\pi_{(g+1)(g+2)} / 2$. Подставляя это выражение в решение $(8)$, получаем потенциал Эрнста в рассматриваемом пределе в терминах отношения тета-функций на $\mathcal{L}$. Можно показать, что это отношение тета-функций на $\mathcal{L}$ выражается через элементарные функции на $\mathcal{L}_{0}$.

Теорема. Потенциал Эрнста (8) с характеристикой (22) в пределе $E_{g+j} \rightarrow$ $F_{g+j}=K_{j}, j=1,2$, тождествен решению Керра, заданному формулой (4), где

$$
e^{-i \varphi}=\frac{i a_{1}+1}{i a_{1}-1} \prod_{i=1}^{g} \sqrt{\frac{K_{1}-F_{i}}{K_{1}-E_{i}}}
$$

ДоКАЗАТЕЛЬСтво. Для доказательства теоремы используем алгебраическую формулировку из предыдушего раздела. Определим дивизор $\widetilde{D}=\xi+\bar{\xi}+\sum_{i=1}^{g} F_{i}$, так что при используемой системе разрезов он будет соответствовать полуцелой характеристике

$$
\frac{1}{2}\left[\begin{array}{lllll}
0 & \cdots & 0 & 1 & 1 \\
1 & \cdots & 1 & 0 & 1
\end{array}\right]
$$


к которой следует прибавить характеристику, соответствуюшую вектору Римана. Отсюда следует, что, как и в разделе 2 , на невырожденной поверхности $\tilde{\mathcal{L}}$ можно определить дивизор $\widetilde{X}=\widetilde{X}_{1}+\widetilde{X}_{2}+X$, где $X-$ дивизор степени $g$, как решение задачи обращения Якоби

$$
\begin{gathered}
\int_{\widetilde{D}}^{\tilde{X}_{1}+\tilde{X}_{2}+X} d \widetilde{\omega}_{g+i}=-i a_{j}, \quad j=1,2, \\
\int_{\widetilde{D}}^{\widetilde{X}_{1}+\widetilde{X}_{2}+X} d \widetilde{\omega}_{i}=0, \quad i=1, \ldots, g .
\end{gathered}
$$

Потенциал Эрнста при этом выражается как

$$
\ln \mathcal{E}=-\int_{\widetilde{D}}^{\tilde{X}_{1}+\widetilde{X}_{2}+X} d \widetilde{\omega}_{\infty^{-} \infty^{+}}
$$

где знак минус возникает из-за того, что характеристика (25) нечетна. В пределе, когда последние два разреза стягиваются, $d \widetilde{\omega}_{i}$ становятся дифференциалами первого рода на $\mathcal{L}$ при $i \leqslant g$, а остальные дифференциалы преврашаются в $d \omega_{K_{i}^{-}} K_{i}^{+}$. Дифференциал $d \widetilde{\omega}_{\infty^{-} \infty^{+}}$принимает вид $d \omega_{\infty^{-} \infty^{+}}$. Поэтому второе уравнение в $(26)$ по теореме Абеля определяет мероморфную функцию (являющуюся рациональной функцией на фундаментальном многоугольнике) с нулями в $\widetilde{X}_{1}+\widetilde{X}_{2}+X$ и полюсами в $D$ :

$$
R(K)=\operatorname{const} \frac{(K-\xi)(K-\bar{\xi}) \prod_{i=1}^{g}\left(K-F_{i}\right)-(A K+B) \mu(K)}{(K-\xi)(K-\bar{\xi}) \prod_{i=1}^{g}\left(K-F_{i}\right)} .
$$

Коэффициенты $A$ и $B$, являющиеся константами относительно $K$, определены тем условием, что нулями функции $R$ являются $X$ и $\widetilde{X}_{i}$. Тогда первое соотношение $(26)$ с учетом (14) есть

$$
-i a_{j}=\frac{R\left(K_{j}^{-}\right)}{R\left(K_{j}^{+}\right)}, j=1,2,
$$

а потенциал Эрнста следует из формуль

$$
\mathcal{E}=\frac{R\left(\infty^{-}\right)}{R\left(\infty^{+}\right)}
$$

Из соотношения (30) с учетом (20) следует, что $\mathcal{G}=-A$. Определяя $A$ из (29), окончательно получаем выражение

$$
A\left(K_{1}-K_{2}\right)=\frac{i a_{1}+1}{i a_{1}-1} r_{1} \prod_{i=1}^{g} \sqrt{\frac{K_{1}-F_{i}}{K_{1}-E_{i}}}-\frac{i a_{2}+1}{i a_{2}-1} r_{2} \prod_{i=1}^{g} \sqrt{\frac{K_{2}-F_{i}}{K_{2}-E_{i}}},
$$

являющееся линейным по $r_{i}$, определенному в (5). В рассматриваемом здесь случае произведения в (31) являются чисто мнимыми, а

$$
\prod_{i=1}^{g} \sqrt{\frac{K_{1}-F_{i}}{K_{1}-E_{i}}} \prod_{i=1}^{g} \sqrt{\frac{K_{2}-F_{i}}{K_{2}-E_{i}}}=1
$$

поскольку $K_{1}=-K_{2}$. Далее, поскольку $a_{1} a_{2}=1$, можно определить $e^{-i \varphi}$ с помощью (24) с вешественным $\varphi$, откуда следует, что

$$
-\mathcal{G}\left(K_{1}-K_{2}\right)=e^{-i \varphi} r_{1}+e^{i \varphi} r_{2} .
$$

А это есть в точности решение Керра в стандартных обозначениях, чем и завершается доказательство. 


\section{4. ЗАКЛЮЧЕНИЕ}

Мы показали, что решение Керра можно получить как частичное вырождение римановых поверхностей высшего рода. Поскольку мы ограничились случаем экваториальной симметрии, мы рассматривали только поверхности четного рода. Это условие можно ослабить, что приводит к так называемым решениям Keppa-NUT, относительно которых, однако, имеется убеждение в их астрофизической несушественности (см., например, [12]). Для того чтобы избежать появления голых сингулярностей, мы также ограничили область изменения физических параметров так, что решения лежат между шварцшильдовским решением и экстремальным решением Керра.

Результат данной работы показывает, каким образом в данный формализм могут быть включены диски материи. В работах [13] диски без центральной черной дыры рассматривались в терминах тета-функциональных решений Короткина. Такие диски можно включить в приведенные здесь формулы по сушеству тем же способом, что и в работе [8]. Поскольку формализм из [8] можно здесь применить с незначительными модифицикациями, то стационарные диски вокруг черной дыры керровского типа можно рассматривать как случай чистого диска.

Благодарности. Автор благодарит Д. Короткина за полезные обсуждения и указания. Работа частично поддержана программой Marie-Curie Европейского Содружества.

\section{Список литературы}

[1] Д. Крамер, Х. Штефани, М. Мак-Каллум, Э. Херльт. Точные решения уравнения Эйншейна. М.: Энергоиздат, 1982.

[2] F. J. Ernst. Phys. Rev. 1968. V. 167. P. 1175; 1968. V. 168. P. 1415.

[3] D. Maison. Phys. Rev. Lett. 1978. V. 41. P. 521.

[4] В. А. Белинский, В. Е. Захаров. ЖЭТФ. 1978. Т. 75. № 6. С. 1955.

[5] Д. А. Короткин. ТМФ. 1988. Т. 77. № 1. С. 25.

[6] Д. А. Короткин, В. Б. Матвеев. Функц. анализ и его прилож. 2000. Т. 34. № 4. С. 18.

[7] C. Klein, O. Richter. Phys. Rev. D. 1998. V. 58. P. 124018.

[8] C. Klein. Phys. Rev. D. 2001. V. 63. P. 064033.

[9] E. D. Belokolos, A. I. Bobenko, V. Z. Enolskii, A. R. Its, V. B. Matveev. Algebro-Geometric Approach to Nonlinear Integrable Equations. Berlin: Springer, 1994.

[10] Д. Мамфорд. Лекции о тета-функциях. М.: Мир, 1988.

[11] J. Fay. Theta-Functions on Riemann Surfaces. Lect. Notes Math. V. 352. Berlin: Springer, 1973.

[12] S. Hawking, G. Ellis. The Large Scale Structure of Space-Time. Cambridge: Cambridge Univ. Press, 1973

[13] C. Klein, O. Richter. Phys. Rev. Lett. 1999. V. 83. P. 2884. 